\title{
DEPARTAMENTO DE MEDICINA CUTÂNEA E INTERNA
}

\section{P044 Associação entre alterações dermatológicas e aterosclerose coronariana em homens: um estudo caso-controle}

\author{
AUTORES: Miot HA, Pandini Filho MA, Gumieiro JH, Cardoso LC, Molina L. \\ INSTITUIÇÃO: Departamento de Dermatologia - FMB - UNESP - Botucatu, SP.
}

FunDMENTOS/OBjetivos: Diversos achados dermatológicos foram associados historicamente à doença coronariana. Alopecia androgenética, distribuição dos pêlos pelo corpo, prega antero-tragal (PAT) e prega longitudinal lobular (PLL) são achados de fácil constatação que podem contribuir na estratificação do risco coronariano.

Material e Métodos: Estudo caso-controle envolvendo pacientes do sexo masculino submetidos a cineangiocoronariografia. Foram considerados como casos aqueles com obstrução de mais de $50 \%$ em pelo menos uma das artérias coronárias. Avaliou-se a presença da PLL, PAT, alopecia e presença de pêlos no tórax nos 2 grupos e os resultados foram ajustados para os fatores de risco: tabagismo, hipertensão, diabetes, idade, dislipidemia e índice de massa corporal.

RESULtados/Dıscussão: Foram analisados 48 pacientes, sendo 36 casos e 12 controles. A prevalência da PLL nos casos $(77,8 \%)$ foi maior que no grupo controle $(33,3 \%)$ (Fisher $p<0,05)$. Alopecia androgenética e distribuição de pêlos no tórax não demonstraram asso ciação positiva com coronariopatia neste estudo (Fisher $p>0,1)$. A ausência da PAT foi significativamente associada a coronárias normais, com uma sensibilidade de 91,7\%, nesse estudo (Fisher $p<0,05$ ). O Odds Ratio (IC95\%), ajustado para os fatores de risco, da PLL foi de $12,6(1,8-85,9)$ e para PAT foi de 50,3 $(3,3-755,9)$ (Regressão Logística Múltipla $p<0,05)$.

Conclusão: Detectou-se associação positiva entre a presença da PLL bilateral e PAT bilateral com aterosclerose coronariana.

\section{PO 45 Mastócitos - Implicação na patogenia do líquen escleroso vulvar?}

AUTORES: Corrêa AC, Azevedo LMS, Almeida GL, Cuzzi T, Takiya CM.
INSTITUIÇÃO: Universidade Federal do Rio de Janeiro - UFRJ - Rio de Janeiro, RJ.

Fundmentos/Objetivos: 0 líquen escleroso (LS) se revela histologicamente com um aspecto característico: presença na derme de uma faixa de colágeno homogênea, hialinizada, abaixo da qual se observa uma faixa de infiltrado inflamatório mononuclear. Essa zona de homogeneização do colágeno serviu de base para uma classificação histopatológica da doença sugerida por Hewitt (1986). No entanto, sua etiologia e fisiopatogenia persistem pouco compreendidas. Em estudo anterior pudemos demonstrar, pelo Azul de Alciano, a presença de mastócitos na derme de pacientes com LS vulvar. O objetivo deste estudo foi quantificar a população mastocitária triptase positiva, presente nos diferentes subtipos histológicos do LS, para verificar a possível influência dos mastócitos na patogenia de tal remodelação da matriz extracelular (MEC).

Material e Ḿ́todos/Casuísnca: Corados pela hematoxilina-eosina, 31 casos de LS vulvar foram subdivididos segundo a gradação histológica de Hewitt. Seguiu-se a histomorfometria dos mastócitos: 1) imunodetecção deste tipo celular por meio da anti-triptase humana (Novocastra); 2) captura das imagens de 10 campos de derme papilar e 10 campos de derme reticular com câmera digital Coolpix 990 (Nikon), utilizando objetiva de 40x; 3) a quantificação foi realizada pelo Image Pro Plus 4.5.1 (USA), programa de análise de imagens. Os resultados foram expressos como o número de mastócitos por área (em $\mathrm{mm}^{2}$ ). Esses resultados foram comparados aos do grupo controle constituído por fragmentos de retalhos cutâneos excisados durante cirurgias corretivas da região vulvoperineal (pele normal, $n=22$ ). Diferenças entre os grupos (e subgrupos) foram verificadas por meio de testes estatísticos.

RESUltados/Dıscussão e Conclusão: O corre redução progressiva da quantidade de mastócitos à medida em que 0 acúmulo de matriz vai se intensificando. A forma ativada deste tipo celular é predominante nos estágios intermediários (Hewitt II.1) da deposição matricial e os mastócitos apresentam tendência a retornar aos níveis basais normais $(p=0,01)$ quando do estágio mais evoluído do acúmulo de MEC (Hewitt grau I.1). Tal comportamento muito se assemelha ao descrito na esclerodermia. Dessa forma, os mastócitos parecem exercer importante papel na remodelação da zona de hialinização do colágeno, contribuindo para sua patogenia como em outras doenças fibrosantes/esclerosantes. 


\title{
P046 Queilite granulomatosa: um estudo clínico, histológico e imunohistoquímico de 22 casos
}

\author{
AUTORES: $\quad$ Lobo AZC, Lourenço SV, Boggio P, Sotto MN, Nico MMS. \\ INSTITUIÇÃO: Departamento de Dermatologia do Hospital das Clínicas da Faculdade de Medicina da \\ Universidade de São Paulo - São Paulo, SP
}

\begin{abstract}
FundAMENTOS: A queilite granulomatosa (QG) é uma apresentação da granulomatose orofacial que pode estar isolada ou associada a outras manifestações clínicas. Clinicamente é caracterizada por edema dos lábios, que inicialmente é transitório, mas que com o tempo se torna permanente. 0 edema labial pode estar associado a edema orofacial (incluindo: pálpebras, mento, glabela, regiões malares, gengiva e mucosa oral). Além disso, a QG pode fazer parte da tríade clássica da síndrome de Melkersson-Rosenthal, juntamente com as manifestações clínicas de paralisia facial e língua plicata.

Oвjetıvos: Revisar a história e as manifestações clínicas e histopatológicas dos doentes com diagnóstico de QG acompanhados na nossa instituição. Avaliar o padrão de citocinas presentes no infiltrado inflamatório das lesões de QG.

Material e Métodos: Encontramos 22 doentes com diagnóstico de QG acompanhados no ambulatório de estomatologia do nosso serviço no período de 1989 a 2004. Os prontuários e as lâminas dos exames histopatológicos foram revisados. Foi feito um estudo imunohistoquímico para a detecção de células expressando TNF-alpha, IFN-gama, IL4 e IL10.

Casuística e Resultados: Entre os 22 casos estudados, 12 eram homens e 10 eram mulheres. A idade no início dos sintomas foi muito variável (dos 10 aos 49 anos de idade), sendo a idade média dos doentes 26,4 anos. Seis $(27,3 \%)$ doentes apresentaram edema facial, além do edema dos lábios. Doze $(54,5 \%)$ apresentavam língua plicata e $5(22,7 \%)$ apresentaram em algum momento paralisia facial. Esses mesmos $5(22,7 \%)$ apresentaram a tríade completa da síndrome de Melkersson-Rosenthal. Os achados histopatológicos variaram desde apenas edema e infiltrado perivascular de linfócitos até formação de granulomas não-necrotizantes. A imunohistoquímica revelou um infiltrado inflamatório com predominância de células expressando TNF-alpha e IFN-gama. 0 tratamento desses doentes se mostrou difícil e as opções mais empregadas foram infiltração com corticóides e drogas anti-inflamatórias como clofazimina e dapsona.
\end{abstract}

Dıscussão E Conclusões: As alterações histológicas na QG não são sempre características, nem fundamentais para o diagnóstico. Esse deve ser baseado na associação da história do doente, características clínicas e respaldado pela histologia compatível. Nossos resultados sugerem que a queilite granulomatosa está associada a um padrão Th1 de citocinas.

\section{PO 47 Psoríase: dados epidemiológicos de ambulatório específico em hospital universitário}

\author{
AUTORES: Miot LDB, Marques SA, Cavalcante PCD, Ribeiro STC, Saad MA. \\ INSTITUIÇÃO: Departamento de Dermatologia - FMB-UNESP - Botucatu, SP
}

Fundamentos/Овjetivos: Psoríase, por ser enfermidade de alta prevalência e potencial de agravos em termos de morbidade clínica e psíquica, faz por merecer acompanhamento diferenciado e o mais personalizado possível. Este ambulatório, ao concentrar os casos de maior complexidade clínico-terapêutica, oferece oportunidades didáticas e de pesquisa. 0 conhecimento dos aspectos epidemiológicos é importante nos planejamentos de saúde loco-regional.

Materials e Métodos: Estudo descritivo envolvendo pacientes com diagnóstico confirmado de psoríase. Por meio de protocolo padrão foram avaliados aspectos relacionados aos pacientes, à doença e à terapêutica.

Resultados/Discussão: Foram analisados 156 pacientes, entre 2003 e 2005. Quanto ao sexo $51 \%$ dos pacientes são femininos, a idade média dos pacientes é de 45,3 anos $+16,1$, a idade de início da doença foi 32,3 anos $+16,5$, ocorreu correlação com história familiar em $26,2 \%$. Houve associação com comorbidades em $57,0 \%$. O uso concomitante de medicamentos foi identificado em $49,3 \%$ dos pacientes; 
uso de bebida alcoólica em $17,9 \%$ e tabagismo detectado em $28,2 \%$. As formas clínicas mais comuns foram a de psoríase vulgar $(73,0 \%)$, seguida por couro cabeludo $(30,1 \%)$ e pela forma eritrodérmica $(12,8 \%)$. Dos pacientes, $56,4 \%$ usavam medicamentos sistêmicos para seu tratamento: $28,8 \%$ metotrexate e $19,8 \%$ acitretina.

ConclusÃo: Os dados apresentados diferem em alguns aspectos clínicos e terapêuticos daqueles da literatura por agregar pacientes de maior complexidade. Saliente-se a oportunidade de demonstração didática aos alunos de graduação dos potenciais de gravidade da psoríase e do treinamento dos médicos residentes em terapêuticas sistêmicas.

\title{
P048 Dermatoses na gravidez: importância do exame dermatológico no pré-natal
}

\author{
AUTORES: Suzuki MM, Pinheiro AM, Suzuki MT, Mosci C, Suzuki AM. \\ INSTITUIÇÃO: Universidade de Ribeirão Preto - Ribeirão Preto, São Paulo
}

\begin{abstract}
FUNDAMENTOS: As alterações cutâneas fisiológicas ou patológicas que ocorrem na gravidez podem estar relacionadas a mudanças metabólicas, endócrinas e imunológicas. O reconhecimento e a classificação dessas manifestações têm importância para seu tratamento e acompanhamento.
\end{abstract}

Овлетіvos: Detectar achados cutâneos por meio de questionário dirigido e exame ectoscópico de gestantes, correlacionando queixas com a presença de dermatoses.

Casuística e Método: Foram atendidas gestantes no período de 24/01/05 a 24/03/05, na Santa Casa, Centros de Saúde I e II de Sertãozinho - SP. As pacientes foram questionadas quanto a queixas cutâneas. Os achados dermatológicos foram classificados em três grupos: alterações fisiológicas na gravidez, dermatoses afetadas pela gravidez e dermatoses específicas da gestação, segundo Krompouzos e cols.

Resultados: 0 total de pacientes atendidas foi 104 , sendo $04(3,8 \%)$ do 10 trimestre, $16(15,3 \%)$ do $2^{\circ}$ trimestre e $84(80,7 \%)$ do 3 o trimestre, com idade entre 14 e 41 anos. Conferimos 33,6\% de gestantes sem queixas dermatológicas, entretanto $100 \%$ delas, após o exame físico, apresentaram algum tipo de dermatose. Encontramos 279 manifestações cutâneas, sendo 254 classificadas como alterações fisiológicas da gravidez, 25 dermatoses afetadas pela gravidez e nenhuma dermatose específica da gestação.

Dıscussão: Observamos, como já comprovado na literatura, que os achados dermatológicos mais freqüentes durante o período gestacional são as modificações fisiológicas e que algumas dermatoses podem ser agravadas ou influenciadas pela gravidez. Não encontramos alterações específicas da gestação, fato relacio nado ao limitado número da amostra. Embora 35 (33,6\%) pacientes não apresentassem queixas dermatológicas, as manifestações cutâneas fisiológicas ou patológicas estavam presentes em $100 \%$ das pacientes.

Conclusão: Dermatoses na gestação são altamente freqüentes, conferindo grande importância ao exame da pele nas consultas de pré-natal.

\section{DEPARTAMENTO DE MICOLOGIA}

\section{PO 49 Levantamento epidemiológico das leveduras oportunistas patogênicas no DF, no período de 2000-2004}

\author{
AUTORES: Pilotto FB, Reis C, Reis Filho E, Theodoro FCET, Silveira VM. \\ INSTITUIÇÃO: Laboratório de Micologia, HUB/UnB - Brasília, DF.
}

INTRODUÇão: Os casos de leveduras oportunistas, principalmente candidíase, têm aumentado recentemente devido ao uso crescente de procedimentos invasivos, drogas e doenças imunossupressoras.

Овлетіvos: Avaliar a prevalência das espécies de leveduras no DF; identificar a distribuição corporal segundo forma clínica e origem do material;estudar o comportamento no sexo e faixa etária.

Casuística/Métodos: Estudo retrospectivo de dados dos livros de registro utilizando protocolo-padrão do Grupo de Estudos do Laboratório de Micologia do HUB (GELM-HUB). Amostra de 1362 exames positivos, de jan/2000 a dez/2004. Raspados cutâneos, fragmentos de unha, secreções, líquidos e biópsias foram corados 